\title{
David Lewis Meets Hamilton and Jacobi
}

\author{
J. Butterfield ${ }^{12}$ \\ All Souls College, University of Oxford \\ (This version submitted 7 Feb 2003)
}

For the PSA02 special issue of Philosophy of Science: Symposium in memory of David Lewis

\begin{abstract}
I commemorate David Lewis by discussing an aspect of modality within analytical mechanics, which is closely related to his work on counterfactuals. This concerns the way Hamilton-Jacobi theory uses ensembles, i.e. sets of possible initial conditions. (A companion paper discusses other aspects of modality in analytical mechanics that are equally related to Lewis' work.)
\end{abstract}

\footnotetext{
${ }^{1}$ Send reprint requests to the author, All Souls College, Oxford OX1 4AL, England.

${ }^{2}$ Many thanks to: Peter Holland and Graeme Segal for conversations; Alexander Afriat, Robert Bishop, Larry Gould, Susan Sterrett and Paul Teller for comments on a previous version; and Gerard Emch and Klaas Landsman for technical help.
} 


\section{Introduction}

It is an honour to take part in commemorating David Lewis. He was a great philosopher, whose genius graced our lives in many ways: how much we miss his transcendent creativity and craftsmanship, his enormous intellectual generosity - and his sense of fun. I propose to commemorate him by discussing modality in analytical mechanics. Though this topic is not close to his interests, it will illustrate a view central to his metaphysical system, and to his influence on analytical philosophy: that science, indeed all our knowledge and belief, is steeped in modality.

Although philosophers seem not to have explored the modal involvements of analytical mechanics, they are both rich and subtle. There is much to explore here: as so often in the philosophy of physics, one can mine from a little physics, a lot of philosophy - at least, a lot more than one paper! To be brief enough, I shall have to be selective in various ways. The three main ones are:-

1) I shall consider only a very limited class of classical mechanical systems, and assume knowledge of how analytical mechanics treats them (Section 2). This limitation is a matter of brevity and convenience: the philosophical discussion in Sections 3 and 4 applies much more widely.

2) Though elementary analytical mechanics is modally involved in many ways, I discuss just one such involvement, which arises in Hamilton-Jacobi theory; for it is closely connected to Lewis' theory of counterfactuals (1973). But several other modal involvements are at least as obvious, and worth pursuing, as this one: e.g. the way the Lagrangian and Hamiltonian approaches' variational principles state the law of motion by mentioning dynamical evolutions that violate the law.

3) This modal involvement, and others, are entangled, technically and philosophically, with the fact that analytical mechanics provides general schemes for solving and representing problems. These general schemes hold philosophical morals, but here I must set them aside. (My (2003, 2003a) discuss several topics which are excluded here by limitations 1) to 3).)

The plan of the paper is as follows. In Section 2, I introduce the elements of analytical mechanics I need. In Section 3, I distinguish three grades of modal involvement, according to which kind of actual matters of fact are varied counterfactually. The rest of the paper concentrates on the first grade, which considers counterfactual initial and/or final conditions, but keeps fixed the forces on the system and the laws of motion. It is strikingly illustrated by Hamilton-Jacobi theory's $S$-function, which represents a structured ensemble of such conditions. The theory involves many different $S$-functions, and so ensembles. So in Section 4, I discuss the structure of this set: in particular, there is an analogy with Lewis' spheres of possible worlds. 


\section{Technical preliminaries}

\subsection{Simple systems; Lagrangian and Hamiltonian mechanics}

I shall consider a mechanical system with $n$ configurational degrees of freedom, whose evolution over time $t$ is given by a curve in a fixed simply connected region $G$ of $(n+1)$ dimensional real space $\mathbb{R}^{n+1}$, on which there are coordinates $\left(q_{1}, \ldots, q_{n}, t\right)=:\left(q_{i}, t\right)=$ : $(q, t)$. If the system consists of $N$ point-particles (or bodies small enough to be treated as point-particles), so that a configuration is fixed by $3 N$ cartesian coordinates, we may yet have $n<3 N$; for the system may be subject to constraints, and the $q_{i}$ are to be independently variable in the region $G$. I shall also assume that the system is simple, in the sense that it has the following six features, (i)-(vi). ${ }^{3}$

Any constraints on the system are to be: (i) scleronomic, i.e. time-independent, so that the region $G$ is a cartesian product of a configuration space $Q \subset \mathbb{R}^{n}$ with a time-interval $\left[t_{-}, t_{+}\right] \subset \mathbb{R}$ (where we allow $t_{-}=-\infty, t_{+}=+\infty$ ); (ii) holonomic; and (iii) ideal. I also assume (iv): The system is to be conservative, and the Lagrangian $L$, defined as the difference of the kinetic energy $T$ and the potential energy $V, L\left(q_{1}, \ldots, q_{n}, \dot{q}_{1}, \ldots, \dot{q}_{n}\right):=T-V$, is to be a $C^{2}$ (twice continuously differentiable) function in all $2 n$ arguments.

These four features yield (the simplest form of) Lagrangian mechanics. For they imply that the laws of motion of the system are given by the Euler-Lagrange (also known as: Lagrange) equations, and that these are equivalent to Hamilton's Principle: that the motion in $Q$ of the point representing the system, between prescribed configurations at times $t_{0}$ and $t_{1}$, makes stationary the action integral $\int L d t$. A curve in $G$ satisfying these laws of motion is called an extremal.

I also assume $(\mathrm{v})$ : that the Hessian of $L$ with respect to the $\dot{q} \mathrm{~s}$ does not vanish in $G$, i.e. the determinant

$$
\left|L_{\dot{q}_{i} \dot{q}_{j}}\right| \neq 0
$$

This yields (the simplest form of) Hamiltonian mechanics, and makes it equivalent to the previous Lagrangian mechanics; as follows. We define canonical momenta, $p_{i}:=L_{\dot{q}_{i}}$; then use eq. 2.1 to solve this definition for the $\dot{q}_{i}$ as functions of $q_{i}, p_{i}, t$ : $\dot{q}_{i}=\dot{q}_{i}\left(q_{j}, p_{j}, t\right)$; then perform a Legendre transformation to introduce the Hamiltonian function and so render the laws of motion, i.e. the Euler-Lagrange equations, equivalent to the canonical (also known as: Hamilton's) equations. Accordingly, we take the system's state-space to be, not $G$ or $Q$, but the $2 n$-dimensional phase space $\Gamma$ coordinatized by the $p$ s and $q \mathrm{~s}$.

Finally, I assume (vi): the region $G \subset \mathbb{R}^{n+1}$ is sufficiently small that between any two "event" points $E_{1}=\left(q_{1 i}, t_{1}\right), E_{2}=\left(q_{2 i}, t_{2}\right)$ there is a unique extremal curve $C$. (I will sometimes suppress the $i$, writing $E_{1}=\left(q_{1}, t_{1}\right), E_{2}=\left(q_{2}, t_{2}\right)$ etc.) This yields (the

\footnotetext{
${ }^{3}$ For further discussion of these features, cf. textbooks such as Arnold (1989) and Lanczos (1986). Some of these features, e.g. (iii), evidently involve modal notions; for discussion, cf. my (2003, 2003a).
} 
simplest form of) Hamilton-Jacobi theory; as follows ...

\subsection{Hamilton-Jacobi theory}

I will describe Hamilton-Jacobi theory in more detail than Lagrangian and Hamiltonian mechanics: for we will need this detail in order to explore its modal involvements. In Section 2.2.1, I introduce the Hamilton-Jacobi equation via Hamilton's characteristic function; then in Section 2.2.2, I discuss hypersurfaces, congruences and fields. Even so, these details will give only a limited view of a very rich theory. In particular:

(1) I will ignore aspects to do with problem-solving (especially the use of separation of variables, leading on to action-angle variables and Liouville's theorem) since - though obviously crucial for physics - they are not illuminating about modality.

(2) I will ignore the integration theory of the Hamilton-Jacobi equation, which involves the theory of generating functions and complete integrals; (though this deep and beautifully geometric theory is illuminating about modality).

(3) Both Sections 2.2.1 and 2.2.2 will emphasise the extended configuration space of Section 2.1, i.e. the region $G \subset \mathbb{R}^{n+1}$; while it is equally illuminating to consider Hamilton-Jacobi theory in phase space. But this emphasis on $G$ will suffice for our purposes - to reveal some distinctive modal involvements.

\subsubsection{The characteristic function and the Hamilton-Jacobi equation}

Assumption (vi) implies that the value of the action integral along the unique extremal $C$ from $E_{1}=\left(q_{1}, t_{1}\right)$ to $E_{2}=\left(q_{2}, t_{2}\right)$ is a function of the coordinates of the end-points. We call this function the characteristic function and write it as

$$
S\left(q_{1}, t_{1} ; q_{2}, t_{2}\right):=\int_{t_{1}}^{t_{2}} L d t=\int_{t_{1}}^{t_{2}}\left(\Sigma_{i} p_{i} \dot{q}_{i}-H\right) d t=\int \Sigma_{i} p_{i} d q_{i}-H d t .
$$

By considering arbitrary small displacements $\left(\delta q_{1}, \delta t_{1}\right),\left(\delta q_{2}, \delta t_{2}\right)$ at $E_{1}, E_{2}$ respectively, one deduces that $S$, considered as a function of the $n+1$ arguments $\left(q_{2}, t_{2}\right)$ (i.e. with $\left(q_{1}, t_{1}\right)$ fixed), obeys the equation-now rewriting $t_{2}, q_{2}$ as $t, q-$

$$
\frac{\partial S}{\partial t}+H\left(q, \frac{\partial S}{\partial q}, t\right)=0
$$

This is the Hamilton-Jacobi equation.

$S$ also defines a family of hypersurfaces, which we can call 'spheres' with centre $E_{1}=\left(q_{1}, t_{1}\right)$ : the sphere around $\left(q_{1}, t_{1}\right)$ with radius $R$ is given by the equation

$$
S\left(q_{1}, t_{1} ; q_{2}, t_{2}\right)=R \text {. }
$$

Every point $E_{2}=\left(q_{2}, t_{2}\right)$ on this sphere is connected to the centre $E_{1}=\left(q_{1}, t_{1}\right)$ by a unique extremal along which the action integral has value $R$. This is amusingly reminiscent of Lewis' spheres of worlds (1973, Chapter 1.3; 1986, Chapter 1.3): and more than amusingly — we will see in Section 4 that the analogy is deeper. 


\subsubsection{Hypersurfaces and fields}

Of course, partial differential equations have many solutions: (the main contrast with ordinary differential equations being that typically, the solution contains an arbitrary function (or functions) rather than an arbitrary constant (or constants)). So HamiltonJacobi theory studies the whole space of solutions of the Hamilton-Jacobi equation. I need to report the main classical result of this study. (For details, a good reference is Rund (1966, Chap. 2), who cites various masters of the last two centuries, especially Carathéodory.) The result connects three diverse notions:-

(a): Families of hypersurfaces in our region $G$ of $\mathbb{R}^{n+1}$

$$
S\left(q_{i}, t\right)=\sigma
$$

with $\sigma \in \mathbb{R}$ the parameter labelling the family; where we assume that $S$ is a $C^{2}$ function in all $n+1$ arguments, and that the family foliates the region $G$ simply in the sense that through each point of $G$ there passes a unique hypersurface in the family.

(b): Congruences of curves that: (i) cross the hypersurfaces and fill $G$ simply in the corresponding sense that through each point of $G$ there passes a unique curve in the congruence; and (ii) may be parametrically represented by $n$ equations giving $q_{i}$ as $C^{2}$ functions of $n$ parameters $u_{\alpha}$ and $t$

$$
q_{i}=q_{i}\left(u_{\alpha}, t\right), i=1, \ldots, n ;
$$

where each set of $n u_{\alpha}=\left(u_{1}, \ldots, u_{n}\right)$ labels a unique curve in the congruence. Such a congruence determines tangent vectors $\left(\dot{q}_{i}, 1\right)$ at each $\left(q_{i}, t\right)$; and thereby also values of the Lagrangian $L\left(q_{i}\left(u_{\alpha}, t\right), \dot{q}_{i}\left(u_{\alpha}, t\right), t\right)$ and of the momentum

$$
p_{i}=p_{i}\left(u_{\alpha}, t\right)=\frac{\partial L}{\partial \dot{q}_{i}} .
$$

(c): Fields, defined to be a set of $2 n C^{2}$ functions $q_{i}, p_{i}$ of $\left(u_{\alpha}, t\right)$ as in eqs 2.6 and 2.7 , i.e. with the $q \mathrm{~s}$ and $p \mathrm{~s}$ related by $p_{i}=\frac{\partial L}{\partial \dot{q}_{i}}$. So a congruence determines a field, and a field determines (by a Legendre transformation, using eq. 2.1) a set of tangent vectors, and so a congruence.

Some jargon: (i) If all the curves of the congruence determined by a field are extremals, the field is called a field of extremals. (ii) We say a field (or its congruence) belongs to a family of hypersurfaces given by eq. 2.5 iff throughout the region $G$ the $p_{i}=\frac{\partial L}{\partial \dot{q}_{i}}$ of the field obey

$$
p_{i}=\frac{\partial}{\partial q_{i}} S\left(q_{i}, t\right)=\frac{\partial}{\partial q_{i}} S\left(q_{i}\left(u_{\alpha}, t\right), t\right) .
$$

(iii) We say that a field $q_{i}=q_{i}\left(u_{\alpha}, t\right), p_{i}=p_{i}\left(u_{\alpha}, t\right)$ is canonical if the $q_{i}, p_{i}$ satisfy Hamilton's equations: equivalently, if the curves of the congruence determined by the field are extremals. 
So much for definitions; now the result. The following three conditions on a $C^{2}$ function $S: G \rightarrow \mathbb{R}$ are equivalent:

(1): $S$ is a $C^{2}$ solution (throughout $G$ ) of the Hamilton-Jacobi equation

$$
\frac{\partial S}{\partial t}+H\left(q, \frac{\partial S}{\partial q}, t\right)=0
$$

(2): The field belonging to the $C^{2}$ function $S: G \rightarrow \mathbb{R}$, i.e. the field defined at each point in $G$ by $p_{i}=\frac{\partial S}{\partial q_{i}}$, is canonical.

(3): The value of the action integral $\int L d t$ along the curve $C$ of the congruence belonging to $S$, from any point $P_{1}$ on the surface $S\left(q_{i}, t\right)=\sigma_{1}$ to that point $P_{2}$ on the surface $S\left(q_{i}, t\right)=\sigma_{2}$ that lies on $C$, is the same for whatever point $P_{1}$ we choose; and the value is just $\sigma_{2}-\sigma_{1}$. That is:

$$
\int_{P_{1}}^{P_{2}} L d t=\sigma_{2}-\sigma_{1}
$$

In the light of eq. 2.10, we call a family of hypersurfaces $S=\sigma$ satisfying any, and so all, of these three conditions geodesically (or: geodetically) equidistant (with respect to the Lagrangian $L)$. So the concentric spheres centred on $E_{1}=\left(q_{1}, t_{1}\right)$ introduced above (eq. 2.4) are an example of a geodesically equidistant family.

This result leaves it an open question which $n$-dimensional surfaces $M$ in $G$ are level surfaces of a $C^{2}$ solution $S$ of the Hamilton-Jacobi equation. In fact it can be shown, subject to some mild conditions about non-vanishing determinants etc., that:

(1): any $n$-dimensional surface $M$ is a level surface of a solution, and this solution is uniquely defined throughout $G$ by its value on $M$ (say $S=0$ on $M$ ); and

(2): for any such surface $M$ and any suitably smooth function $S: M \rightarrow \mathbb{R}$, there is a uniquely defined solution on all of $G$ which restricts on $M$ to the given $S$. (So (2) generalizes (1) by $M$ not having to be a level surface.)

In the jargon: the initial value problem for the Hamilton-Jacobi equation has locally a solution, that is unique given suitably smooth prescribed values of $S$. But I shall not go into details about this. It suffices to state the intuitive idea for the case where $M$ is a level surface. The solution is "grown" from the given surface by erecting a congruence of curves, transverse to the surface, and passing along them to mark off a given value of the action integral $\int L d t$. By varying the value, one defines a geodesically equidistant family and so a solution $S$.

Returning finally to mechanics: it is clear that each solution $S$ of the HamiltonJacobi equation represents a kind of ensemble, i.e. a fictitious population of mechanical systems (maybe including the actual system). Thus each solution $S$ represents an ensemble with the feature that at all times $t$, there is a strict configuration-momentum, i.e. $q-p$, correlation given by the gradient of $S$. That is, $S$ prescribes for any given $(q, t)$, a unique $(p, t):=\left(\frac{\partial S}{\partial q}, t\right)$.

So much by way of expounding Hamilton-Jacobi theory. I will return, after Section 3 's introduction of modality, to discuss the structure of this set of ensembles (set 
of $S$-functions) - and so the modal involvements of Hamilton-Jacobi theory. I end this Section by emphasising that, as mentioned in Section 1, my restriction to simple systems is a matter of brevity and expository convenience, not of substance. Much of the formalism above, and the philosophical morals below, apply much more widely.

\section{Grades of modal involvement}

In the light of Section 2, I think it is natural to distinguish (in Quinean fashion!) three grades of modal involvement in analytical mechanics. So I shall write (Modality;1st) etc. Like Quine's three grades, the first is intuitively the mildest grade, and the third the strongest. But this order will not correspond to Section 2's (and the historical) order of the three approaches to analytical mechanics: Lagrangian, Hamiltonian and Hamilton-Jacobi. In particular, the first and arguably most intuitive approach, Lagrangian mechanics, exhibits the third grade of modal involvement.

The grades are defined in terms of the kind of actual matters of fact they allow to vary counterfactually. ${ }^{4}$ The first kind is, roughly, the state of the system. The second kind is the physical problem: which we can take as specified by the number of degrees of freedom, and the Lagrangian or Hamiltonian which encodes all the forces on the system. A third kind is the laws of motion, as specified by e.g. Hamilton's Principle or Lagrange's or Hamilton's equations. Thus we have the following grades.

(Modality;1st): The first and mildest grade keeps fixed the actual physical problem and laws of motion. But it considers different initial conditions and/or final conditions than the actual ones. And so it also considers counterfactual histories of the system; (since under determinism, a different initial or final state implies a different history, i.e. trajectory in state-space).

This grade is evident throughout analytical mechanics. It arises from the postulation of a state-space, be it (in Section 2's notation) $Q$ or $\Gamma$ or $G$. For example, recall from our definition of a simple system: (i) the configuration space $Q$ is to have independently variable coordinates $q_{i}$; and (ii) to define ideal constraints, one needs the notion of a virtual displacement, i.e. a displacement that the system could undergo compatibly with the constraints and applied forces. But the most striking illustration of (Modality;1st) is Hamilton-Jacobi theory; cf. Section 4.

(Modality;2nd): The second grade keeps fixed the laws of motion, but considers different problems than the actual one (and thereby different initial states). For example, it considers a counterfactual number of degrees of freedom, or a counterfactual potential function. Maybe no actual system is a simple system with 5,217 coordinates; or with a potential given (in certain units) by the polynomial $13 x^{7}+5 x^{3}+42$. But

${ }^{4}$ I of course set aside the (apparent!) fact that the actual world is quantum, not classical; so I talk about e.g. an actual system obeying Hamilton's Principle. Since my business throughout is the philosophy of classical mechanics, it is unnecessary to encumber my argument with antecedents like 'If the world were not quantum': I leave you to take them in your stride. 
analytical mechanics continually considers such counterfactual cases: in Section 2, we generalized from the outset about the number $n$ of degrees of freedom, and about what the Lagrangian or Hamiltonian was. Such generality of course pays off in general theorems.

(Modality;3rd): The third grade considers different laws of motion, even for a given problem. This occurs even in the Lagrangian and Hamiltonian mechanics of simple systems; namely in their use of Hamilton's Principle. (And we saw in Section 2.2 that these variational principles are also involved in the Hamilton-Jacobi approach.) In all three approaches, the use of variational principles means - not that one explicitly states non-actual laws, much less calculates with them-but that one states the actual law by comparing the actual history of the system with counterfactual histories that do not obey the law. This is at first sight surprising, even mysterious. How can it be possible to state the actual law by a comparison of the actual history with possible histories that do not obey it? In particular, many philosophers hold that any actual truth is made true by an actual "truthmaker": a principle which such a statement of the laws of motion apparently violates. I take up these questions in Butterfield (2003).

To sum up, analytical mechanics gives many illustrations of all three grades: the subject is upto its ears in modality. But rather than multiplying examples, the remainder of this paper focusses on Hamilton-Jacobi theory's illustration of (Modality;1st). There is no special philosophical difficulty here: rather the situation presents an invitation to philosophers to study a new sort of modal structure.

\section{On the set of ensembles}

Since the $S$-function, representing an ensemble of systems whose $q$ and $p$ are correlated by $p=\frac{\partial S}{\partial q}$, stands at the centre of Hamilton-Jacobi theory, it is clear that the theory provides (Modality;1st) in spades. I stress that this use of ensembles involves no suspicious "possibilia power" (Lewis 1986a, p. 158). That is, there is no strange influence (whether causal or constitutive) of the $S$-function, or the ensemble it represents, on the actual system (or propositions about it). In particular, the evolution of a system (its trajectory in configuration space or phase space) is fixed by, for example, the initial conditions $-q, \dot{q}$ in Lagrangian mechanics and $q, p$ in Hamiltonian mechanics - irrespective of which if any $S$-function we care to use. ${ }^{5}$

As mentioned at the end of Section 2.2, the structure of the set of ensembles (set of $S$-functions) is essentially given by the structure of the set of suitably smooth (say $C^{2}$ )

\footnotetext{
${ }^{5}$ I say 'care to use' since, as mentioned at the start of Section 2.2, S-functions are principally used to solve otherwise intractable mechanical problems. Incidentally, the situation is different in quantum theory. There, $S$ has a close mathematical cousin (also written $S$ ) whose values do influence the motion of the system. But again, this does not involve any weird "possibilia power". For this influence is regarded as a strong, indeed the strongest, reason to take the quantum $S$-function as part of the actual physical state of the individual system; i.e. not as in classical mechanics, as "just" a description of an ensemble.
} 
real functions on a $n$-dimensional manifold $M ;(M$ needs to "lie across" the region $G$ so as to be transverse to a congruence of extremals). For since there is a locally unique solution to the Hamilton-Jacobi initial value problem, each such function determinesas well as is determined by - a solution throughout $G$ of the Hamilton-Jacobi equation. So one infers that the set of solutions (ensembles) is some kind of infinite-dimensional set.

This set has various kinds of structure, and a full discussion would take us into those aspects of Hamilton-Jacobi theory that we had to set aside at the outset of Section 2.2. But even with just the results of Section 2.2, we can discern two kinds of structure - which bear on Lewis' account of counterfactuals and modality. These two kinds of structure arise from two different choices about what to take as the analogue, in Hamilton-Jacobi theory, of a Lewisian possible world.

\subsection{Configurations as worlds}

Let us think of an event (i.e. instantaneous configuration) $\left(q_{i}, t\right) \in G$ as like a possible world. Then Hamilton's characteristic function eq. 2.2, and the geodesic spheres it defines eq. 2.4, yield a neat analogy with Lewis' theory of counterfactuals. For recall Lewis' proposed truth-conditions for a counterfactual 'If $A$ were the case, then $C$ would be the case', which I will write as $A \rightarrow C$ (1973, Chap. 1.3). Lewis wants to avoid the assumption that there is a set of $A$-worlds all tied for first equal as regards similarity to the actual world @ (the Limit Assumption). He also allows the counterfactual to be vacuously true: namely iff no world in the union of nested spheres around @, $\cup \$ @$, makes $A$ true. So Lewis proposes that the counterfactual $A \rightarrow C$ is true at @ iff:-

1) no $A$-world belongs to any sphere $S$ in the system $\$ @$ of spheres around @;

or

2) some sphere $S$ in the system $\$ @$ contains at least one $A$-world, and $A \supset C$ is true at every world in $S$; (i.e. $C$ is true at every $A$-world in $S$ ).

We can easily transplant this kind of truth-condition to geodesic spheres; i.e. taking points $\left(q_{i}, t\right) \in G$ as worlds and $\int L d t$ as the measure of distance (dissimilarity) between such worlds. However, the resulting conditionals hardly deserve the name 'counterfactual', since both the "base-world" $\left(q_{1}, t_{1}\right)$ and the "closest $A$-world", say $(q, t)$, that the evaluation of the conditional carries us to, could be actual configurations of the system. For simplicity I will ignore the vacuous case, 1) above. This yields the following truth-condition, relative to a given configuration $\left(q_{1}, t_{1}\right)$ :

a) $A$ is true at a possible configuration $(q, t)$, to which the given configuration $\left(q_{1}, t_{1}\right)$ could evolve (i.e. would evolve for some $p_{1}$ at $\left.t_{1}\right)$ with $t>t_{1}$; and

b) for every possible configuration $\left(q^{\prime}, t^{\prime}\right)$ to which $\left(q_{1}, t_{1}\right)$ could evolve with $t>t_{1}$, and such that $\int_{q_{1}, t_{1}}^{q^{\prime}, t^{\prime}} L d t \leq \int_{q_{1}, t_{1}}^{q, t} L d t$ :

$A \supset C$ is true at $\left(q^{\prime}, t^{\prime}\right)$; (i.e. if $A$ is true at $\left(q^{\prime}, t^{\prime}\right)$, so is $C$ ). 
In the abstract, this truth-condition seems a mouthful. But in fact mechanics provides countless examples of such conditional propositions, though of course in a much less formal guise! A very simple example is given by a bead sliding on a wire that lies in a vertical plane; (to be a simple system, the bead must slide frictionlessly). We can take $A$ to say that the bead is at the lowest point of the wire, and $C$ to say that its potential energy is at a minimum. Then $A \rightarrow C$ can be expressed informally as 'Whenever the bead is next at the lowest point of the wire, its potential energy will then be at a minimum'. Similarly, with $C$ saying instead that the kinetic energy is at a maximum; and so on.

Finally, the results in Section 2.2.2 (especially condition (3)) implies that this discussion of counterfactuals can be generalized so as to define similarity of worlds using $S$-functions other than Hamilton's characteristic function. For example, we could take a $n$-dimensional surface $M$ that is topologically a sphere surrounding some given point $\left(q_{1}, t_{1}\right) \in G$, define $M$ to be a surface of constant $S$, say $S=0$, and consider the (locally unique) solution of the Hamilton-Jacobi equation thereby defined outside $M$. That is, we could define the dissimilarity of our worlds $(q, t)$ from the base-world $\left(q_{1}, t_{1}\right)$, and so the truth-conditions of counterfactuals, in terms of the value of $S(q, t)$.

\subsection{States as worlds}

On the other hand, let us take as the analogue of a Lewisian world an instantaneous state in the sense of a $2 n+1$-tuple $\left(q_{i}, p_{i}, t\right)$. This is perhaps a more natural choice than Section 4.1's instantaneous configurations (events), since it determines a history, i.e. a phase space trajectory, of the system, our "toy-universe". There are various constructions one could make with this concept of world. In particular, one could define conditionals $A \rightarrow C$ by using a solution $S$ of the Hamilton-Jacobi equation to define dissimilarity. But for the sake of variety, I shall not pursue this. I shall instead describe how an $S$-function enables us to define various sets of possible worlds which are "preferred" relative to our choice of $S$; in fact, the last of these definitions is important for physics.

Here again, the $S$-function can be any solution of the Hamilton-Jacobi equation. Given such an $S$, every point $(q, t) \in G$ has an associated canonical momentum, viz.

$p:=\frac{\partial}{\partial q} S(q, t)$, and so an associated world in our sense, viz. $\left(q, p \equiv \frac{\partial S}{\partial q}, t\right)$. If we wish, we can also pick out subsets so that not every event $(q, t)$ is included in a world "preferred" by our $S$. For example, we could do this by picking out a sub-manifold $M$ of $G$, and defining the associated worlds $\left(q, p \equiv \frac{\partial S}{\partial q}, t\right)$ only for $(q, t) \in M$.

There are two obvious ways to specify such an $M$; both make $M n$-dimensional. First, we can define $M$ as the level surface of $S$ that passes through some given $(q, t) \in G$. This definition will connect $M$ with Section 2.2.2's discussion of geodesically equidistant hypersurfaces. And thinking of $(q, t)$ as the system's actual present configuration, $M$ defines a preferred set of counterfactual events, i.e. instantaneous configurations (which are in general not simultaneous with $(q, t)$ ). 
Secondly, we can fix $t$. For the chosen $t$, we consider the gradient $\frac{\partial}{\partial q} S(q, t)$ of $S$ as a function on $Q$. The preferred worlds are then given by all $\left(q, p \equiv \frac{\partial}{\partial q} S(q, t)\right)$ for $q \in Q$. So the worlds are given as before, except that the fixed value of $t$ is now implicit in the definition of $p$.

This definition gives us a suitable note to end on. For it turns out that this second definition is crucially important for the mathematics and physics of Hamilton-Jacobi theory in phase space. It leads to the mathematics of Lagrangian submanifolds, and the physics of focussing and caustics (and even to the quantum-classical relation!). In fact, we can analyse the structure of the set of possible preferred sets by studying the set of all Lagrangian manifolds; (for some more details, cf. e.g. Arnold (1989, Chap.s $7,8)$ ). So I like to think of Lewis' genius - always so creative, insightful and generousgiving us a philosophical perspective on the deep and beautiful structures of classical mechanics.

\section{References}

Arnold, V. (1989), Mathematical Methods of Classical Mechanics, New York: SpringerVerlag (second edition).

Butterfield, J. (2003), "Some Aspects of Modality in Analytical Mechanics", forthcoming in Formal Teleology and Causality, ed. M. Stöltzner, P. Weingartner, Paderborn: Mentis.

Butterfield, J. (2003a), "Postulating all States, Solving all Problems: some philosophical morals of analytical mechanics". In preparation.

Lanczos, C. (1986), The Variational Principles of Mechanics, New York: Dover (4th edition).

Lewis, D. (1973), Counterfactuals, Oxford: Blackwell.

Lewis, D. (1986), On the Plurality of Worlds, Oxford: Blackwell.

Lewis, D. (1986a), Philosophical Papers, volume I, New York: Oxford University Press.

Rund, H. (1966), The Hamilton-Jacobi Theory in the Calculus of Variations, New York: Van Nostrand. 